\title{
On the response of ultra microbalances to inkjet dispensing of fluids
}

\author{
Hans-Jochen Trost \\ MicroFab Technologies, Inc. \\ Plano, Texas, U.S.A. \\ E-mail: hans-jochen.trost@microfab.com
}

\begin{abstract}
Inkjet gravimetry has become a useful method for controlled fluid dispensing. The microbalances used for weighing the fluid are designed for static, unchanging weights, so their application under continuously changing load due to more or less slow fluid accumulation and/or evaporation needs a modified approach to evaluating the weight data reported by the balance. In the present work, models for both fluid accumulation and evaporation are outlined and examined, and rules for obtaining reliable measurements of dispensed fluid amounts derived.
\end{abstract}

Keywords: inkjet, microbalance, gravimetry, evaporation

\section{Introduction}

Inkjet gravimetry has been used in recent years for improving the control of inkjet dispensing amounts in terms of mass or volume $[1,2,3,4,5,6,7,8]$ and carried further into applications like the manufacturing of test samples for detection of explosive or narcotic substances [9, 10, 11, 12, 13], study of nanomaterial properties [14] and controlled fluid injection in capillary electrophoresis [15]. The concept is to integrate a microbalance with an industrial inkjet printer and use dispensing of a predetermined number of droplets into a weighing boat on the balance to determine the average droplet mass and/or, if the density is known, the droplet volume. If, in addition, the composition of the fluid is uniform and well known, the mass measurement can serve to control the use of any fluid ingredient of interest. Similarly, gravimetry and a density meter have been integrated on other fluid dispensing systems for calibration purposes [16, 17].

When dispensed into a weighing boat, the fluid to be weighed is by necessity exposed to air and thus will evaporate. The resulting continuous weight change prevents the microbalance from ever reaching a stable reading. This brings the time dependence of the response by the balance to a given load into play, which is particularly critical when the rate of load change varies, like at the beginning and end of a period of dispensing fluid into the weighing boat. The objective of the present study is to quantify the time dependent effects of evaporation and balance response to come up with workable rules for reliable and reproducible measurements of droplet masses.

In the present study, an ultra microbalance is used for weighing, and three types of experiments are performed: First, a set of calibration weights is measured repeatedly to establish the response behavior of the balance to a well-defined weight for different operational settings of the balance. Second, evaporation of three different fluids is measured with varying environ- mental conditions to establish a quantitative model. For these two exercises, a desktop setup is used. Third, droplet mass measurements are performed on an inkjet printer to help outline an empirical model for the time dependence of the response behavior of the balance.

\section{Experimental setup}

The gravimetry for the present work is performed with an ultra microbalance model XP2U by Mettler-Toledo (Greifensee, Switzerland), controlled through a server program developed for the jetlab 4 XLB print station [18] of MicroFab Technologies, Inc. (Plano, Texas, USA). The program can also be used for stand-alone data recording. In order to limit the rate of evaporation, the regular draft shield is removed and replaced with a custom-made much smaller cylindrical shield with an interior air volume of $13.7 \mathrm{~mL}$ and with a $3 \mathrm{~mm}$ diameter hole in the top, through which the fluid dispensing into a small weighing boat on the weighing pan is possible (see figure S1 in the Supplementary Information and figure 9 in [10]). Smooth-walled cylindrical tin capsules with flat bottom, $8 \mathrm{~mm}$ in diameter and $12 \mathrm{~mm}$ high (item D4094, Elemental Microanalysis, Okehampton, Devon, UK), are used as weighing boats.

The settings of the XP2U include among others a mode selection for different styles of weighing and an environment setting by which different degrees of filtering are applied to adapt to vibrations imparted onto the weighing system. As the environment is different for different installations where inkjet gravimetry is used, it is important to assess the effect of these settings. As an example, 100 seconds worth of data for evaporation of water, recorded at a rate of approximately $2.3 \mathrm{~Hz}$, are shown in figure 1 for three combinations of the mode and environment settings. "Universal" mode and "Standard" environment are the factory defaults for the weighing application; "Sensor" mode includes various degrees of filtering controlled 
by the environment setting, of which the "Standard" and "Unstable" options are considered in the present study. As can be seen in figure 1, the filtering smoothes out the balance response quite significantly. The unsteady behavior of being slow to follow weight changes and catching up suddenly every approximately 5 seconds has been observed by the author consistently for the half dozen balances he has worked with, and the data displayed by Liang et al. [5] show clearly the same effect, despite having their equipment in an enclosure located on an optical table with good vibration isolation. This behavior is also not unique to this manufacturer and model.

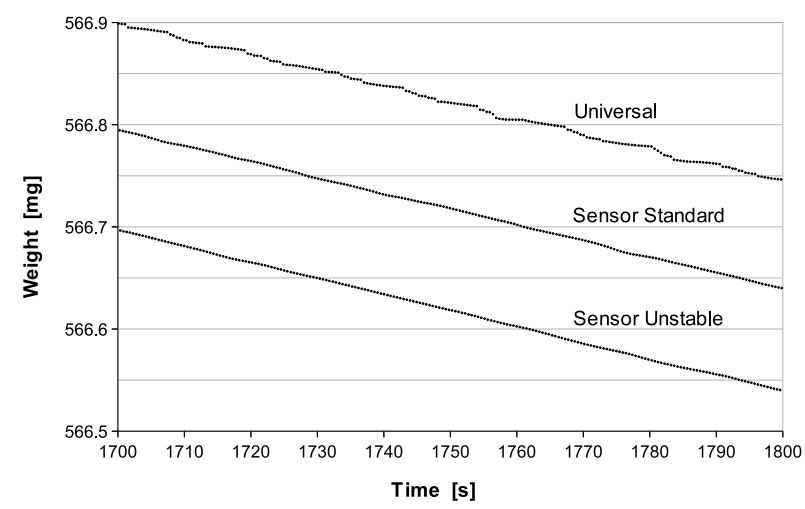

Figure 1: Evaporation of water recorded every $0.4 s$ over $100 s$. Data were recorded successively for the three mode and environment settings and shifted in time and weight for easier comparison.

Starting with this core equipment, three sets of experiments were performed: 1) repeated static measurements of calibration weights, 2) measurement of evaporation rates of water, ethanol and isobutanol under varying conditions, and 3) measurement of the mass of water droplets using varying sizes of aliquots.

For the static weight measurements, calibration weights of $10 \mathrm{mg}, 20 \mathrm{mg}, 50 \mathrm{mg}$ and $200 \mathrm{mg}$, OIML class F1, were used (Troemner, Thorofare, New Jersey, USA). The regular draft shield of the ultra microbalance was left in place, with the top cover on but permanently opened to one side for the frequent loading and unloading.

For the evaporation measurements, the balance was used without the regular shield. Data were acquired both without and with the small draft shield in place, and with air blown across the pan at two different speeds to obtain data for an evaporation model that includes the effect of air draft above the fluid surface. This is of interest because inkjet printers may include scrubbers pulling air out of the machine enclosure or HEPA filters blowing air in. This experiment was set up on a desk, with a 6 inch diameter fan (personal fan model F-0621, Optimus Enterprise, Inc., Anaheim, California, USA) placed $50 \mathrm{~cm}$ away from the weighing pan. The fan was placed on an independent support, not on the desk together with the balance, thus preventing vibrations from the fan to reach the balance. In order to avoid possible sliding of the boat off of the pan due to the air stream hitting directly on the full side of the boat, a square block was set between the fan and balance with a flat top at about $2 \mathrm{~mm}$ below the rim of the boat. Wind speeds were measured both above and behind the boat using a handheld anemometer (model PMA85, Pyle Audio, Inc., Brooklyn, New York, USA) with a nominal threshold and resolution of $0.1 \mathrm{~m} / \mathrm{s}$, range to $30 \mathrm{~m} / \mathrm{s}$ and advertised uncertainty of $\pm 5 \%$. The fan produced two speeds, about $0.6 \mathrm{~m} / \mathrm{s}$ and $0.8 \mathrm{~m} / \mathrm{s}$ at the boat. For the present work, one and a half times the resolution is used for the uncertainty as $5 \%$ of the air velocities of interest is well below the readout resolution. Temperature and humidity were monitored about half a meter away from the balance with a hygro-thermometer (model no. 445703, Extech Instruments, Nashua, New Hampshire, USA) with accuracies $\pm 5 \% R H$ and $\pm 1{ }^{\circ} \mathrm{C}$. The local barometric pressure, not corrected to sea level, was observed with a barometer integrated in a sports watch (Outbreaker model RP107, Oregon Scientific, Tualatin, Oregon, USA) with 0.01 inch of mercury resolution. While some of the equipment is to be rated consumer grade rather than industrial, it is adequate for the present purposes. For reference, weather data recorded by the National Weather Service at three local airports (Addison, McKinney, Rockwall) near Plano, Texas were retrieved over time from their web pages.

The dispensing measurements were performed on a jetlab 4 XLB [18] placed on a sturdy lab table. The standing feet of the print station provide a modest amount of vibration isolation. During balance measurements, the control program of the printer turns off power to the motors of the motion stages, thus removing that potential source of vibration. The balance is always used in its converted configuration with the small draft shield.

The instant weight data from the balance are read via RS232C as fast as the hardware will allow; this turns out to be a rate of approximately $5 \mathrm{~Hz}$. The server program keeps the data in an internal cyclical buffer sufficiently large for some 6 hours worth of data. Two data streams can be directed to hard disk. One usually receives a data point $0.4 \mathrm{~s}$, the other $10 \mathrm{~s}$, after its predecessor is written, leading to rates a little slower than $2.5 \mathrm{~Hz}$ and $0.1 \mathrm{~Hz}$ respectively. The second stream also receives results from fits to droplet mass measurements, including the unabridged data used in analyzing for the mass. The time data are recorded and stored in milliseconds since 1 Jan 1970 0:00 o'clock local time, and the weight data to full resolution of $0.1 \mu \mathrm{g}$. In evaluating measurement uncertainties, variously also called measurement errors, single standard deviation values are used, corresponding to the $k=1$ coverage factor defined in the Guide to the Expression of Uncertainty in Measurement (GUM [19]). The extended uncertainties with coverage factors $k=2$ and $k=3$ then translate into two and three standard deviation errors.

\section{Analysis methods}

The measurement method for static masses as well as fluid aliquots is the burst method described, among others, in references $[1,4,5]$. The weight data $w_{i}$ at the times $t_{i}$ returned by the balance before loading of the test mass, be it a solid weight or a fluid aliquot, as well as after loading and expiration of a 
grace period letting the balance catch up to the load change, are fit by linear expressions (2).

$$
\begin{array}{r}
f\left(\mathbf{a} ; t_{i}\right)=a_{0}+a_{1} \times t_{i} \\
\min _{\mathbf{a}} \sqrt{\sum_{i=1}^{n}\left(w_{i}-f\left(\mathbf{a} ; t_{i}\right)\right)^{2}}
\end{array}
$$

In the case of fluid mass measurements, the weighing boat is pre-filled with fluid to a level covering the bottom completely, in order to maintain a constant surface for evaporation over time, and the system is given time to saturate the interior volume of the draft shield. Under these conditions, evaporation is slow, still not negligible, and for all practical purposes linear over time. (There is a small dependence on the level height in the weighing boat.) The time intervals used are called in the following discussions the lead and tail intervals. The resulting straight lines (1) are extrapolated towards each other to a time $t_{c}$ and the difference between the two yields the desired mass value

$$
m=f_{\text {tail }}\left(t_{c}\right)-f_{\text {lead }}\left(t_{c}\right)
$$

The uncertainty from the extrapolation is evaluated through prediction intervals $\pm \delta_{\text {prd }}[20,21]$

$$
\delta_{p r d}^{2}=t_{1-\alpha / 2 ; n-p}^{2} \sigma_{e v}^{2}\left(\mathbf{x}_{c}^{T}\left[X^{T} X\right]^{-1} \mathbf{x}_{c}\right)
$$

with $t_{1-\alpha / 2 ; n-p}$ being the $1-\alpha / 2$ quantile of the Student distribution for $n-p$ degrees of freedom ( $p=2$ in the present case), where for a $k=1$ uncertainty $\alpha=0.6834$ is applied. The uncertainty $\sigma_{e v}$ of the individual weight data points, assumed equal for all of them, is estimated through equation (5).

$$
\sigma_{e v}^{2}=\frac{1}{n-p} \sum_{i=1}^{n}\left(w_{i}-f_{\text {bestfit }}\left(\mathbf{a} ; t_{i}\right)\right)^{2}
$$

Further, $\mathbf{x}_{c}$ is a $p$-vector of partial derivatives at the time $t_{c}$

$$
\mathbf{x}_{c}=\left(\partial f\left(\mathbf{a} ; t_{c}\right) / \partial a_{j}\right), \quad j=1, \ldots, p
$$

and $X$ is a $n \times p$ matrix of partial derivatives at the fitted weight data points

$$
X_{i j}=\partial f\left(\mathbf{a} ; t_{i}\right) / \partial a_{j} \quad i=1, \ldots, n ; j=1, \ldots, p
$$

For the static mass measurements, this is by far the dominant uncertainty. The fluid aliquots require more terms to be taken into account. First, a buoyancy correction is applied:

$$
\begin{aligned}
m= & \left(f_{\text {tail }}\left(t_{c}\right)-f_{\text {lead }}\left(t_{c}\right)\right) \times B\left(\rho_{\text {fluid }}\right) \\
B\left(\rho_{\text {fluid }}\right)= & \left(\rho_{\text {steel }}-\rho_{\text {air }}\right) \times \rho_{\text {fluid }} / \\
& \left(\rho_{\text {steel }} \times\left(\rho_{\text {fluid }}-\rho_{\text {air }}\right)\right)
\end{aligned}
$$

Here the densities $\rho$ apply to the dispensed fluid (with relative uncertainty 0.0015$)$, the steel of the internal calibration weight (fixed at $8000 \pm 200 \mathrm{~kg} / \mathrm{m}^{3}$ ) and air (fixed at $1.2 \pm 0.2 \mathrm{~kg} / \mathrm{m}^{3}$ ). Then its uncertainty as well as the various manufacturerspecified uncertainties are added in quadrature to those from the extrapolations:

$$
\delta_{m}^{2}=\delta_{\text {prd,lead }}^{2}+\delta_{\text {prd,tail }}^{2}+\delta_{\text {buoy }}^{2}+\delta_{\text {th }}^{2}+\delta_{\text {rnd }}^{2}+\delta_{\text {rep }}^{2}+\delta_{\text {lin }}^{2}
$$

For the XP2U, the last four terms are $\delta_{t h}=1.0 \times 10^{-6}{ }^{\circ} C^{-1} \times$ $5^{\circ} \mathrm{C} \times m$ for the thermal effect of $5^{\circ} \mathrm{C}, \delta_{r n d}=0.05 \mu \mathrm{g}$ for the rounding from the readout resolution, $\delta_{\text {rep }}=0.25 \mu \mathrm{g}$ for the repeatability, and $\delta_{\text {lin }}=0.9 \mu \mathrm{g}$ for the linearity.

The data of the individual burst weight measurements, in particular for the larger bursts, can be used to model the response by the balance to a weight change over time. The advantage here over the manually conducted static mass measurements is that, due to the computer control of the print station on which the data were taken, there is a fairly good control over and knowledge of the timing of events, in particular the start and stop times of the dispensing.

The simplest approach to a finite response is to use an exponential saturation function (10).

$$
m(t)=m_{0}\left(1-e^{-\lambda t}\right)
$$

This equation applies to weighing of a mass $m_{0}$ that appears on the balance instantaneously at the time $t=0 \mathrm{~s}$. In practice, the mass is accumulated at a constant rate $R=m_{0} / T_{0}$ over a time interval of length $T_{0}$. Thus, for any given time $t$, weight contributions have to be integrated over the past time of accumulation (11).

$$
m(t)=R \int_{0}^{T(t)} 1-e^{-\lambda(t-u)} d u
$$

The upper integration limit is the current time while the accumulation is going on, and the length of the accumulation interval thereafter, $T(t)=\min \left(t, T_{0}\right)$. A transformation of variables gives an integral that is known analytically (12).

$$
\begin{array}{r}
m(t)=R \int_{0}^{T(t)} d u+R \int_{t}^{t-T(t)} e^{-\lambda x} d x \\
=R T(t)-\frac{R}{\lambda}\left[e^{-\lambda x}\right]_{t}^{t-T(t)}
\end{array}
$$

The first term $(R T(t))$ represents the accumulated mass on the balance, and the second the lag effect of the response of the balance to the presence of that mass. The method of Brodlie [22] is used to fit the total weight over time reported by the balance (13)

$$
w(t)=m(t)+W_{0}-W_{\text {evap }} t
$$

to the data in the least-squares sense with constant weights. The same time range as for the extrapolation method is used, and the evaporation offset $W_{0}$, evaporation rate $W_{\text {evap }}$, dispensed total mass $m_{0}$ and response coefficient $\lambda$ are varied in the fit. It turns out that the response function needs to be shifted in time by a small amount $t_{s}$, which could easily be explained by the control program and controller firmware taking some time between inquiring the computer clock for the start time for the dispensing and the actual onset of pulsing at the output of the controller. It also helps, in a crude way, to accommodate the shape change of the response introduced by the filtering in sensor mode operation.

$$
w(t)=m\left(t-t_{s}\right)+W_{0}-W_{\text {evap }} t
$$

With this extra parameter added to the fit, a generally quite satisfactory description of the data is obtained (figures 2,3 ). 

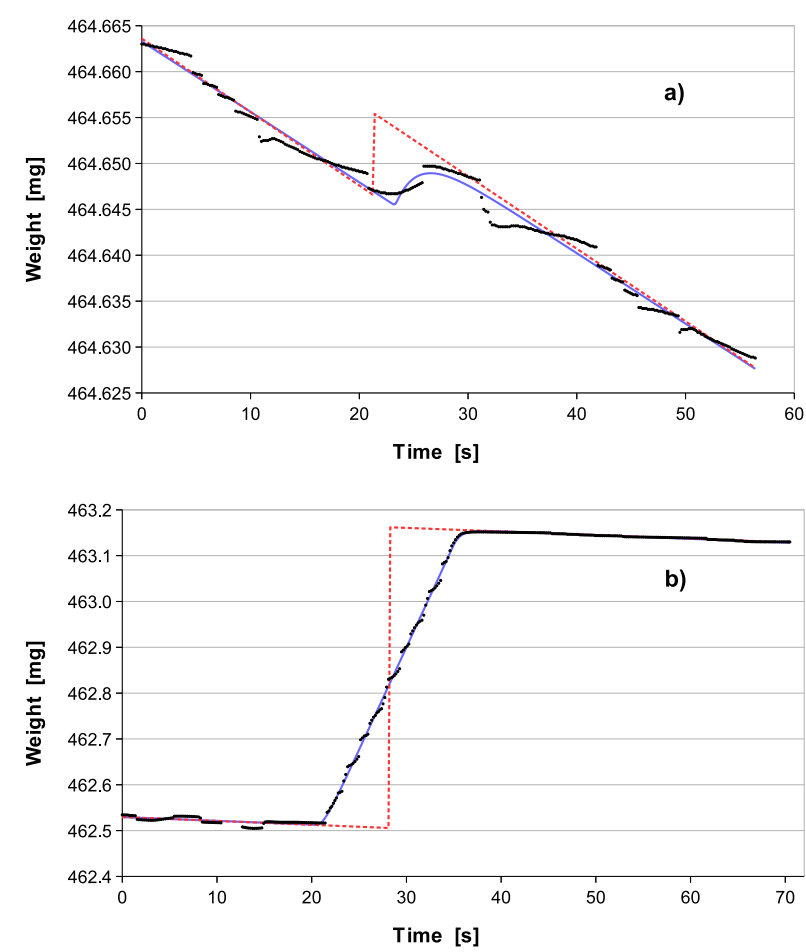

Figure 2: Example fits for Universal/Standard settings. a) 50 droplets; b) 5000 droplets. The time line includes $20 s$ for evaporation control, $1 s$ buffer time, $0.3 s$ and $14 s$ respectively for dispensing at $500 \mathrm{~Hz}$ including control overhead, $15 s$ wait time for balance response, and $20 s$ for evaporation control. Red dashed lines show the extrapolation result using equation (3) with the step up located at time $t_{c}$, blue solid lines the curves using equation (14).

The uncertainty in the droplet mass, which is derived from the dispensed mass $m_{0}$ found by fitting equation (14) to the data and the known number of dispensed droplets, is determined with an algorithm following the general concept of the MINOS processor in the MINUIT optimization package of the CERN Program Library [23]. The mass $m_{0}$ is multiplied by 0.9 and the other four parameters in equation (14) are freely readjusted to fit the data. If the resulting increase of $\chi^{2}$ is smaller than 1 unit, the new mass value is reduced further by $0.9^{2}$ and the test repeated. Further steps, if needed, use increasing powers of 0.9 . Once this chain of fits exceeds the $\chi^{2}$ criterion, the last step is replaced by a reduction by 0.93 and the chain resumes with this base factor. Further iterations use $0.99,0.993$ and 0.999 . At that point, a linear interpolation step terminates the search for $m_{\text {low }}$ which gives a fit resulting in $\chi_{\text {low }}^{2}=\chi_{\text {min }}^{2}+1$. The same exercise using division by the same scale factors instead of multiplication yields a value $m_{h i g h}$ with $\chi_{\text {high }}^{2}=\chi_{\min }^{2}+1$. The desired uncertainty is then

$$
\delta m_{0}=\max \left(m_{0}-m_{\text {low }}, m_{\text {high }}-m_{0}\right)
$$

The needed $k=1$ uncertainty $\sigma$ is assumed to be the same for all weight data values and is estimated from the optimal fit using equation (5) (with $w_{\text {best } f i t}(t)$ taking the place of $f(\mathbf{a} ; t)$ ).
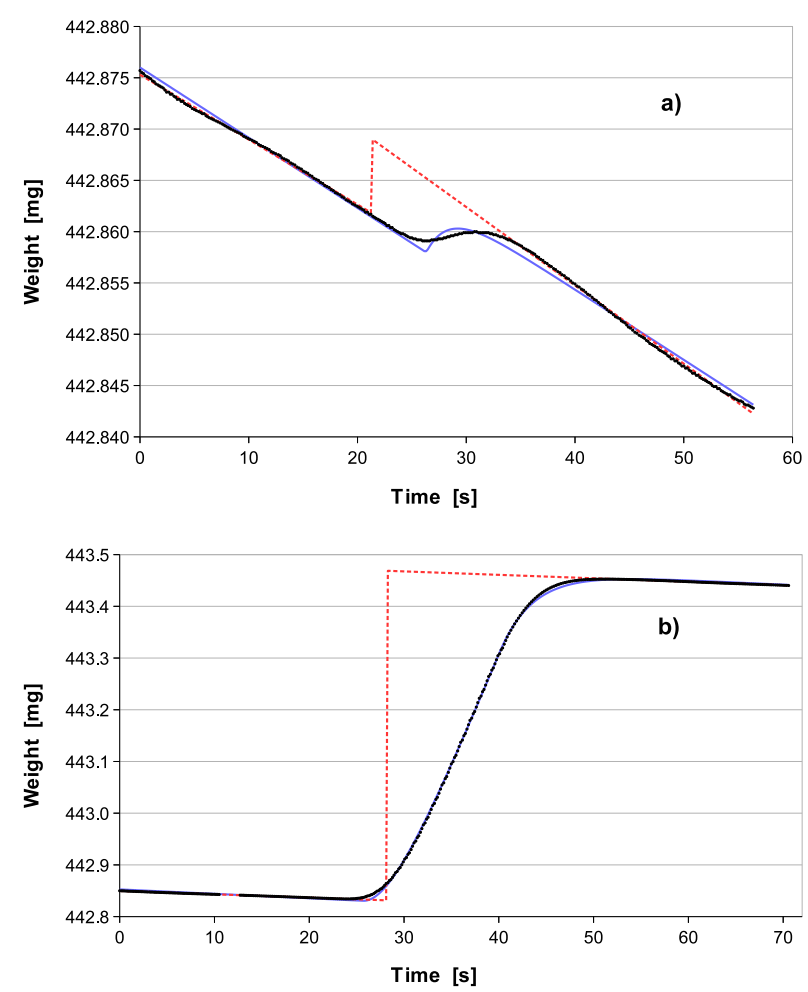

Figure 3: Example fits for Sensor/Unstable settings. a) 50 droplets; b) 5000 droplets. The same time line and mark-up as in figure 2 is used.

\section{Data acquisition and results}

\subsection{Static mass measurement}

The measurement is started with an internal calibration of the balance, followed by setting the readout to zero. The calibration weights were placed with tweezers onto the weighing pan, removed a minute later, loaded again another minute later, continuing in this manner until all data were taken. The choice of one minute was found to ensure stable readings for at least $25 \mathrm{~s}$ between loading and unloading for any operating conditions under consideration. Each weight was loaded 8 times, and the full series was performed for each of the three filter settings of the balance described above (figure S2 in the Supplementary Information). Visual inspection of the raw weight data (figure 4) suggests a response time of less than the manufacturer's specification of $10 \mathrm{~s}$. Mass and response time data were derived as follows: A window of 50 consecutive data points (approximately $22 s$ long) was fit with a straight line, and the window moved point by point through the data. All fits with an absolute value of the slope larger than $2.5 \times 10^{-4} \mathrm{mg} / \mathrm{s}$ ("loose cut") were discarded. For each load event, the last retained line before loading and the first retained line after loading were combined to calculate the mass and the response time of the balance. Arbitrarily assigning a load period of $1 s$ at the beginning of the response, the algorithm used for the burst method of measuring dispensed fluid aliquots $[1,4,5]$ was applied, i.e., extrapolation from both lines to the center of the load period. The response time was 


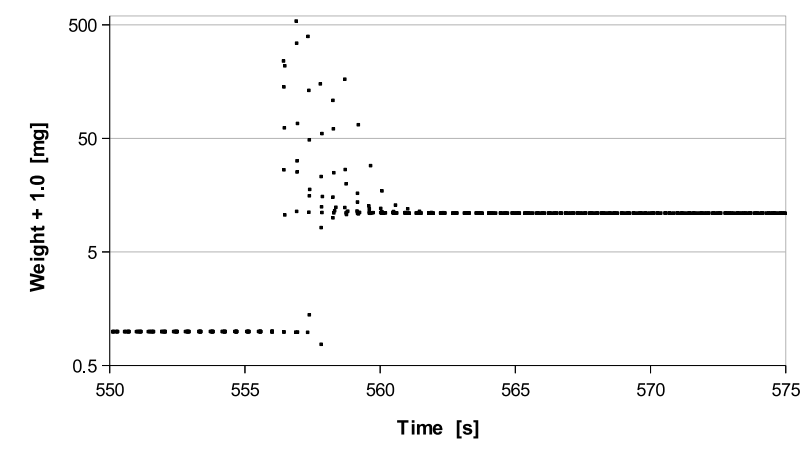

Figure 4: Overlay of weight data for all 8 measurements of $10 \mathrm{mg}$ with Universal/Standard settings. Data are shifted up $1 \mathrm{mg}$ to allow for a logarithmic scale. The repeat measurements are synchronized at $556.0 \mathrm{~s}$, using the last data point in each case with $\left|w_{i}\right|<10 \mu \mathrm{g}$.

Table 1: Balance response times from fixed mass measurements, loose slope cut.

\begin{tabular}{cccc}
\hline Weight & Universal/Std. & Sensor/Std. & Sensor/Unstable \\
\hline $10 m g$ & $5.5 \pm 2.7 s$ & $11.5 \pm 2.4 s$ & $19.6 \pm 3.1 s$ \\
$20 \mathrm{mg}$ & $5.1 \pm 1.6 \mathrm{~s}$ & $13.2 \pm 1.2 \mathrm{~s}$ & $20.6 \pm 2.0 \mathrm{~s}$ \\
$50 \mathrm{mg}$ & $7.8 \pm 4.8 s$ & $12.3 \pm 1.5 \mathrm{~s}$ & $26.0 \pm 6.1 \mathrm{~s}$ \\
$200 \mathrm{mg}$ & $9.6 \pm 4.9 \mathrm{~s}$ & $14.7 \pm 4.2 \mathrm{~s}$ & $26.6 \pm 5.6 \mathrm{~s}$ \\
\hline
\end{tabular}

taken to be the elapsed time between the last point in the line before loading the weight and the first point in the line after.

The results are shown in table 1 , with the errors being just the statistical ones for the number of repeated measurements. Tables 2 and 3 show the same for slope cuts at $1 \times 10^{-4} \mathrm{mg} / \mathrm{s}$ ("tight cut") and $5 \times 10^{-5} \mathrm{mg} / \mathrm{s}$ ("stringent cut") respectively, which leads naturally to successively larger response time values. The corresponding mass results are compiled in tables S1-S3 in the Supplementary Information, with the uncertainties summarized in table 4. They are satisfactory given the rough environmental conditions (no vibration isolation, open draft shield, manual loading and unloading) and focus on estimating a reasonable response time rather than on obtaining the best possible weight measurements. The increased uncertainties for the filtered data are believed to be due to the algorithm not knowing the exact load time (first contact of the weight with the weighing pan) nor the actual response time. The mass results for the loose slope cut are expected to be worst of the three cases, as the tables indeed show.

Table 2: Balance response times from fixed mass measurements, tight slope cut.

\begin{tabular}{cccc}
\hline Weight & Universal/Std. & Sensor/Std. & Sensor/Unstable \\
\hline $10 m g$ & $11.0 \pm 5.7 s$ & $15.6 \pm 3.4 s$ & $22.1 \pm 4.1 s$ \\
$20 \mathrm{mg}$ & $11.2 \pm 4.2 s$ & $18.7 \pm 3.7 s$ & $27.9 \pm 8.3 \mathrm{~s}$ \\
$50 \mathrm{mg}$ & $10.7 \pm 5.6 \mathrm{~s}$ & $15.4 \pm 2.8 \mathrm{~s}$ & $30.3 \pm 7.0 \mathrm{~s}$ \\
$200 \mathrm{mg}$ & $13.0 \pm 6.7 \mathrm{~s}$ & $17.7 \pm 5.7 \mathrm{~s}$ & $30.0 \pm 7.8 \mathrm{~s}$ \\
\hline
\end{tabular}

Table 3: Balance response times from fixed mass measurements, stringent slope cut.

\begin{tabular}{cccc}
\hline Weight & Universal/Std. & Sensor/Std. & Sensor/Unstable \\
\hline $10 m g$ & $15.1 \pm 5.5 s$ & $18.6 \pm 4.3 s$ & $26.6 \pm 5.5 s$ \\
$20 \mathrm{mg}$ & $17.1 \pm 11.2 s$ & $21.3 \pm 5.6 s$ & $32.1 \pm 14.7 s$ \\
$50 \mathrm{mg}$ & $12.9 \pm 5.3 s$ & $21.9 \pm 6.0 s$ & $37.6 \pm 11.7 s$ \\
$200 \mathrm{mg}$ & $14.9 \pm 7.6 s$ & $21.6 \pm 7.9 s$ & $33.7 \pm 12.0 s$ \\
\hline
\end{tabular}

Table 4: Uncertainties of fixed mass measurements

\begin{tabular}{cccc}
\hline Cut & Universal/Std. & Sensor/Std. & Sensor/Unstable \\
\hline loose & $\pm 0.25 \mathrm{mg}$ & $\pm 0.82 \mathrm{mg}$ & $\pm 1.50 \mathrm{mg}$ \\
tight & $\pm 0.10 \mathrm{mg}$ & $\pm 0.41 \mathrm{mg}$ & $\pm 0.58 \mathrm{mg}$ \\
stringent & $\pm 0.06 \mathrm{mg}$ & $\pm 0.18 \mathrm{mg}$ & $\pm 0.25 \mathrm{mg}$ \\
\hline
\end{tabular}

\subsection{Evaporation measurement}

Three fluids were used: deionized water, ethanol and isobutanol. Data were acquired for the first two on one day and water and isobutanol three days later, with the repetition of water serving to check for comparable experimental conditions. Each data set consists of two parts, one with the weighing boat completely open to ambient air, and the second with the draft shield cap placed over the boat. Each part, in turn, is broken into 5 sections: fan off, fan at lower speed, fan at higher speed, fan at lower speed and fan off. The observable of interest is the evaporation rate. By averaging the two fan-off and the two slowfan periods respectively, comparison to the fast-fan data will have the dependence of the evaporation rate on the height of the fluid level removed to first order. Each data set starts with a nearly full weighing boat and consumes a sufficient fraction of the contained fluid that at the end a slowing effect in the evaporation rate becomes evident, due to the cylindrical wall now limiting the opening angle for the fresh vapor to diffuse away from the liquid surface. As an example, the raw weighing data for ethanol are shown in figure 5. The evaporation rates for the corresponding sections of measurement are collected in table 5.

Among a number of model descriptions for evaporation rates, only the one proposed by Carrier [24, 25] appears to treat the cases of no air draft and finite velocity air draft across the surface on an equal footing and is used here in the form of equation (16).

$$
\begin{aligned}
-d W / d t & =A\left(P_{\text {sat }}-P_{\text {dew }}\right)\left(c_{0}+c_{v} v_{\text {air }}\right) / \Delta H_{v} \\
& =r_{0}+r_{v} v_{\text {air }}
\end{aligned}
$$

Here, $A=50.3 \mathrm{~mm}^{2}$ is the surface area of the fluid (set by the cross section of the boat), $P_{\text {sat }}$ is the saturation vapor pressure at

Table 5: Evaporation rates for ethanol for five sections of the data in figure 5a.

\begin{tabular}{ccc}
\hline Wind speed & open & under cap \\
\hline $0.0 \mathrm{~m} / \mathrm{s}$ & $25.073 \pm 0.150 \mu \mathrm{g} / \mathrm{s}$ & $6.559 \pm 0.004 \mu \mathrm{g} / \mathrm{s}$ \\
$0.6 \mathrm{~m} / \mathrm{s}$ & $123.554 \pm 0.477 \mu \mathrm{g} / \mathrm{s}$ & $13.631 \pm 0.036 \mu \mathrm{g} / \mathrm{s}$ \\
$0.8 \mathrm{~m} / \mathrm{s}$ & $126.375 \pm 0.516 \mu \mathrm{g} / \mathrm{s}$ & $13.068 \pm 0.043 \mu \mathrm{g} / \mathrm{s}$ \\
$0.6 \mathrm{~m} / \mathrm{s}$ & $98.911 \pm 1.050 \mu \mathrm{g} / \mathrm{s}$ & $11.840 \pm 0.036 \mu \mathrm{g} / \mathrm{s}$ \\
$0.0 \mathrm{~m} / \mathrm{s}$ & $6.572 \pm 0.003 \mu \mathrm{g} / \mathrm{s}$ & $5.354 \pm 0.004 \mu \mathrm{g} / \mathrm{s}$ \\
\hline
\end{tabular}



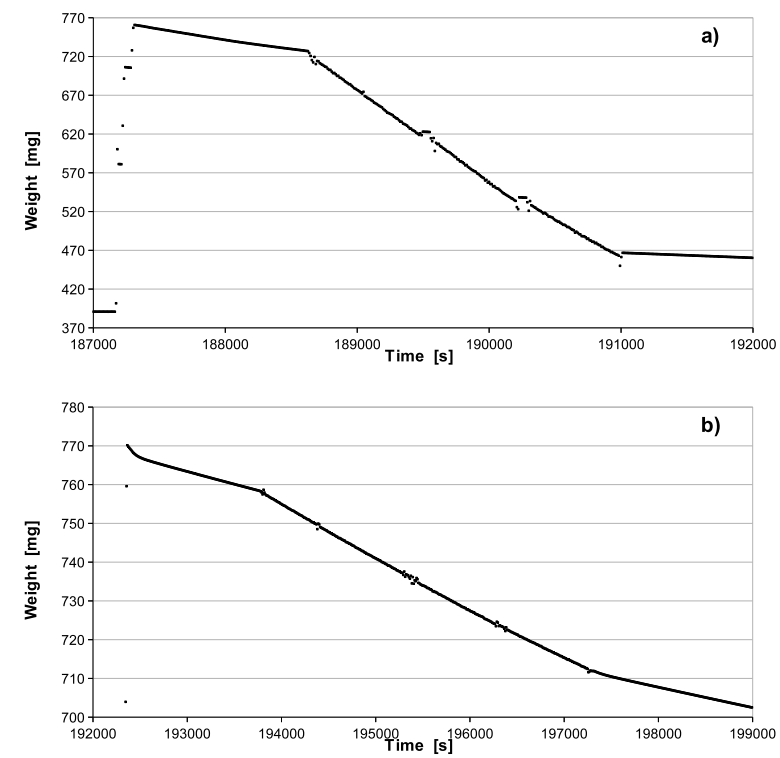

Figure 5: Raw weight data for evaporation of ethanol - a) weighing boat open to air; b) weighing boat covered by draft cap. For analysis, $600 s$ long sections of apparently constant evaporation rate were used, avoiding the visible curvatures in the covered case and disturbances from shaking of the setup. The rise at the left end of the diagrams represents the filling of the boat, starting completely empty in the open case.

Table 6: Fitted coefficients of evaporation rates (see text section 4.2).

\begin{tabular}{lcc}
\hline & $r_{0}[\mu \mathrm{g} / \mathrm{s}]$ & $r_{v}[\mu \mathrm{g} / \mathrm{m}]$ \\
\hline Data of 5 Feb 2016 & & \\
Water, open & $4.37 \pm 0.03$ & $27.42 \pm 6.11$ \\
Water, cap & $1.50 \pm 0.003$ & $1.65 \pm 0.46$ \\
Ethanol, open & $15.86 \pm 2.10$ & $144.61 \pm 35.31$ \\
Ethanol, cap & $5.96 \pm 0.17$ & $10.39 \pm 2.38$ \\
Data of 8 Feb 2016 & & \\
Water, open & $4.45 \pm 0.20$ & $25.00 \pm 5.98$ \\
Water, cap & $1.34 \pm 0.11$ & $2.06 \pm 0.46$ \\
Isobutanol, open & $4.33 \pm 3.48$ & $48.39 \pm 10.44$ \\
Isobutanol, cap & $1.07 \pm 0.04$ & $1.42 \pm 0.29$ \\
\hline
\end{tabular}

the current temperature (typically $23^{\circ} \mathrm{C}$ during the present experiments), $P_{\text {dew }}$ is the partial vapor pressure given the relative humidity (typically $24 \%$ during the present experiments), $\Delta H_{v}$ is the latent heat of evaporation of the fluid, and $v_{\text {air }}$ the velocity of the air streaming across the fluid surface. The coefficients $c_{0}$ and $c_{v}$ are treated as adjustable parameters and are derived from directly fitting $r_{0}$ and $r_{v}$. The data for ethanol and the results of fitting all data are collected in tables 5-7 and figure 6 .

The fits of the velocity dependence were performed using Brodlie's derivate-free convex optimization algorithm [22]. The parameter uncertainties attributable to the fitting were determined by a grid search for the largest deviation of either parameter, with no restriction on the respective other one, constrained by an increase of the target function $\chi^{2}$ by 1 . Here, $\chi^{2}$ is defined by equation (17)

$$
\chi^{2}=\sum_{i=1}^{N}\left(R_{i}-\left(r_{0}+r_{v} v_{i}\right)\right)^{2} / \sigma_{i}^{2}
$$
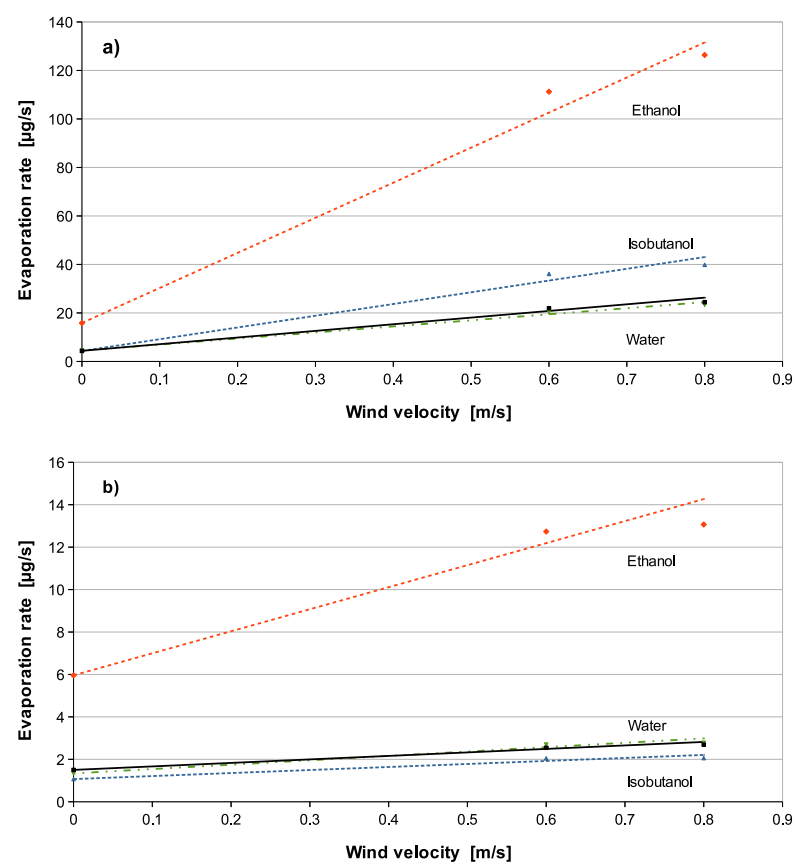

Figure 6: Evaporation rates as a function of the velocity of air flowing across the setup - a) weighing boat open to air; b) weighing boat covered by draft cap.

with $N=3$ counting the wind velocities used, $R_{i}$ being the rate of weight loss due to evaporation at the wind velocity $v_{i}$ and $\sigma_{i}$ being the measured $k=1$ uncertainty of $R_{i}$.

The quoted uncertainties for $r_{0}$ and $r_{v}$ include those from the fit to the evaporation rates at the three wind velocities $(0.0 \mathrm{~m} / \mathrm{s}$, $0.6 \mathrm{~m} / \mathrm{s}$ and $0.8 \mathrm{~m} / \mathrm{s}$ ) weighted by the respective uncertainties of the rates and a contribution of the uncertainty of the measured wind velocity. The impact of the latter on the overall uncertainty was estimated as follows: With the readability of the anemometer at $0.1 \mathrm{~m} / \mathrm{s}$ being larger than the manufacturer's quoted uncertainty of $5 \%$, an uncertainty of $0.15 \mathrm{~m} / \mathrm{s}$ was used. Further, as the measured velocities for the fast and slow settings came out always at $0.1-0.3 \mathrm{~m} / \mathrm{s}$ apart (with the average value of $0.2 \mathrm{~m} / \mathrm{s}$ most of the time), the uncertainty was considered to be dominantly systematic (i.e., a type B uncertainty in the terminology of the GUM [19]). The propagation of this uncertainty into the velocity dependence represented in equation (16) was therefore obtained by repeating the fits with the velocity values changed to $0.0,0.45$ and $0.65 \mathrm{~m} / \mathrm{s}$ for one side and $0.0,0.75$ and $0.95 \mathrm{~m} / \mathrm{s}$ for the other side. As an example, the primary fit for the case of ethanol open to air gives parameter uncertainties of $0.074 \mu \mathrm{g} / \mathrm{s}$ and $0.541 \mu \mathrm{g} / \mathrm{m}$. The lower velocities shift the parameters by $-2.095 \mu \mathrm{g} / \mathrm{s}$ and $-35.308 \mu \mathrm{g} / \mathrm{m}$, while the higher velocities give changes of $-0.548 \mu \mathrm{g} / \mathrm{s}$ and $24.752 \mu \mathrm{g} / \mathrm{m}$. This domination of the total uncertainty by the ones for the wind velocity appears in all present cases. In the uncertainties of the coefficients $c_{0}$ and $c_{v}$, the wind velocity is responsible for one half to two thirds of their uncertainties. For the contributions from the external parameters, $A, P_{\text {sat }}, P_{\text {dew }}$ and $\Delta H_{V}$, conservative estimates were used: The area may be uncertain to $10 \%$ due 
Table 7: External and fitted parameters for equation 16.

\begin{tabular}{lccccc}
\hline & $P_{\text {sat }}[\mathrm{kPa}]$ & $P_{\text {dew }}[\mathrm{kPa}]$ & $\Delta H_{v}[\mathrm{~kJ} / \mathrm{kg}]$ & $c_{0}\left[\mathrm{~m}^{-1} \mathrm{~s}^{-1}\right]$ & $c_{v}\left[\mathrm{~m}^{-2}\right]$ \\
\hline Water, open & 2.93 & 0.67 & 2330 & $0.090 \pm 0.024$ & $0.563 \pm 0.194$ \\
Ethanol, open & 5.33 & 0 & 846 & $0.050 \pm 0.014$ & $0.456 \pm 0.158$ \\
Water, open & 2.93 & 0.67 & 2330 & $0.091 \pm 0.024$ & $0.514 \pm 0.182$ \\
Isobutanol, open & 1.15 & 0 & 579 & $0.043 \pm 0.036$ & $0.484 \pm 0.158$ \\
\hline
\end{tabular}

to deformation of the thin-walled boat, the saturated vapor pressures also to $10 \%$ mainly due to inaccuracy of the environmental temperature used, the dew point pressure includes $10 \%$ each for temperature and humidity uncertainty, and the latent heat of evaporation $20 \%$ from the scattering of the published data. Further uncertainties due to environmental conditions are expected to be negligible as their measurement, as well as the repetition of the experiment with water, showed them to be fairly constant during the data acquisition (the comparison to the weather data is included in the Supplementary Information). This does not imply that a change of environmental conditions would have no effect on the measurements.

For the original model application of evaporation from swimming pools $[24,25], c_{0}=0.089 \mathrm{~m}^{-1} \mathrm{~s}^{-1}$ and $c_{v}=0.0782 \mathrm{~m}^{-2}$ are frequently quoted, with $c_{0}$ showing a close agreement with the present experiment. As the rim of the weighing boat stands somewhat higher up above the fluid surface in a different proportion than the typical edge of a swimming pool, any agreement between parameters may be considered more of a surprise than any difference.

In terms of the inkjet gravimetry, the main lesson is that evaporation over time can be treated with a linear model even in the presence of a continuous air draft. The surface area for evaporation and the humidity or partial vapor pressure above the surface have to be kept constant over time. This last condition is apparent from the raw data in figure $5 \mathrm{~b}$, where short periods showing curvature in the time dependence indicate the buildup of a saturated atmosphere under the shield: at the beginning of the data taking, when the draft shield was first placed over the weighing boat, and after turning the fan off, when the draft ceases to pull humid air through the hole in the top of the shield.

It has been demonstrated [26] that adding a layer of slowevaporating liquid (a silicone oil in that case) will essentially block the evaporation of the dispensed fluid as it collects in the weighing boat, reducing the related uncertainties in the mass measurement. However, this trick will often not be available because no suitable layer fluid is available (e.g., for isobutanol or acetonitrile $[12,27])$. Also, it exposes dispensed droplets to a higher evaporation rate in flight (discussed further below near the end of section 4.3) due to the lower saturation of the air with vapor, an effect of increasing importance with decreasing droplet size. The present data (table 6) show differences between the evaporation rates below the cap and open to air by a factor of 3-4. According to the Carrier model (16) this translates into a proportional change of the vapor pressure and the vaporized fluid volume under the cap. Full saturation of the $13.7 \mathrm{~mL}$ under the cap by water (vapor density of $23 \mathrm{~g} / \mathrm{m}^{3}$ at $25^{\circ} \mathrm{C}$ ) would take $315 \mu \mathrm{g}$ of water or nearly 2900 droplets of
$60 \mu m$ diameter. With the expected evaporation loss in flight in the order of below one to a very few percent, mass measurements would need to involve very high numbers of drops dispensed in a limited time to significantly raise the local humidity. Typical applications by users of jetlab 4 XLB systems that the author is aware of measure a few thousands of droplets at a time, so the humidity will not increase noticeably and the evaporation rate in unsaturated air will apply.

\subsection{Droplet mass measurement}

The gravimetric droplet mass measurements for the present study reproduce in concept the measurement by Verkouteren and Verkouteren [1] of the effect of the aliquot size. A sequence of burst mode measurements of different aliquot sizes was defined (in the order 2000, 50, 5000, 200, 20, 10000, 500, 1000,100 droplets) and data were acquired a total of 10 times through that cycle. The dispensing frequency was set to $500 \mathrm{~Hz}$, well below the range where the droplet mass is significantly affected [2, 28]. Due to burst mode limitations in the dispensing controller, bursts larger than 500 droplets were broken into sub-bursts of 500 droplets, with the breaks between sub-bursts lasting a fraction of a second each. The 10 cycles were split in half and bracketed by continuous mode measurements of 5000 droplets 13 times in a row. Of the 13 repetitions lasting $10 \mathrm{~s}$ each, the first 3 were discarded to allow the balance response to adjust to the net rate of weight change during dispensing and the remaining 10 were averaged. In terms of equation (12), the discarding of $30 s$ worth of data accounts safely for the response lag time even when using the Sensor mode with the "Unstable" filter setting. The continuous mode data provide a reference statistically similar to the burst mode data but free of problems with the initial droplets in a burst [1], and a simple check on the stability of the dispensing throughout the whole experiment.

Examples of individual fits for bursts of 50 and 5000 droplets are displayed in figures 2 and 3 for both the extrapolation and analytical response function approaches of equations (3) and (14). The raw burst mass data, sorted by size of the aliquot, are collected in figure 7 and the derived droplet masses in figure 8 . The impact of the different operating modes of the microbalance is obvious, and despite the positive effect of the filtering, a reasonably accurate measurement of the droplet mass appears to require aliquots of at least 500 droplets, and preferably a few thousand. Taking the average uncertainty of the individual measurements (figure 7, i.e., $2.22 \mu \mathrm{g}$ and $1.31 \mu \mathrm{g}$ for the Universal and Sensor modes respectively) and asking for a $k=2$ extended uncertainty of $1 \%[1,2]$ leads to a requirement of at least 3670 and 2040 droplets (of $128 \mathrm{ng}$ ). These numbers are much larger than those derived using the rules of United States 

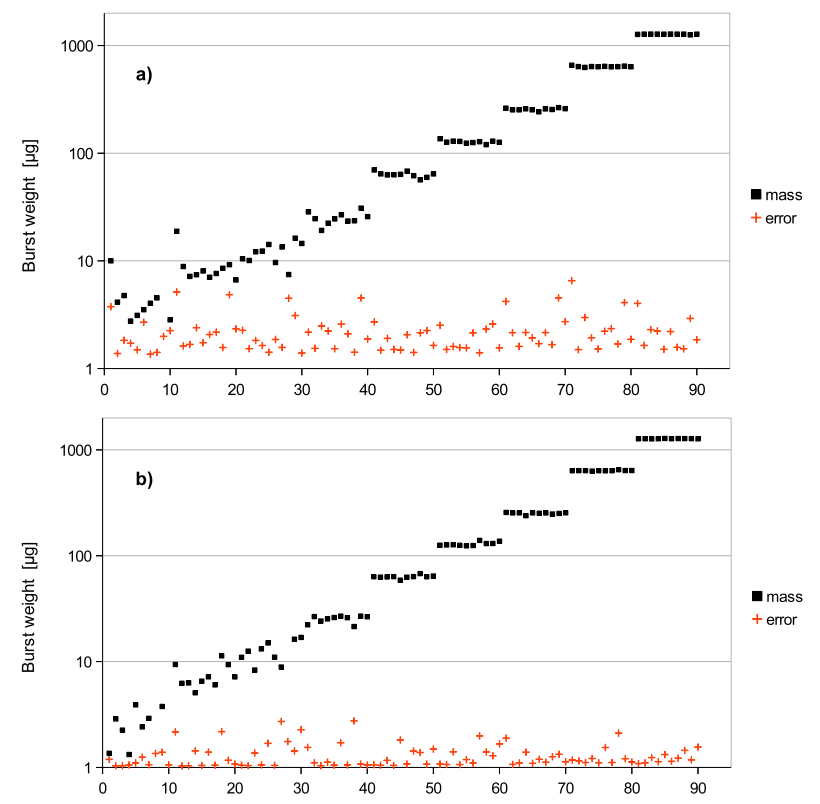

Figure 7: Raw burst weight data from two-sided extrapolation sorted by aliquot size - a) Universal/Standard settings; b) Sensor/Unstable settings.

Pharmacopeial Convention ("USP") [29] for setting a minimum weight above which balance measurements are considered accurate. The USP rule is $2 \sigma_{\text {rep }} / m \leq 0.10 \%$, which with the manufacturer's specification of the repeatability of $\sigma_{\text {rep }}=0.25 \mu \mathrm{g}$ translates for the XP2U into 3910 droplets (note the $0.10 \%$ versus $1 \%$ criteria). ${ }^{1}$ The difference is likely due both to USP's reliance on the repeatability as the dominant uncertainty and the comparatively rough experimental environment in the present work. If smaller aliquots are of interest, the division into subbursts can be taken advantage of; e.g., to measure 50 droplets one might work with a total burst of about or above 2000 in subbursts of 50. This approach has been discussed in some detail by Verkouteren and Verkouteren [1, 2].

The individual response fit results for the burst mass and resulting averaged droplet masses are displayed in figures 9 and 10. While the response fit of equation (14) is more restrictive on the evaporation background in forcing the evaporation rate to be constant throughout the measurement (the extrapolation method allows it to be different before and after dispensing), it actually has five variable parameters, whereas the extrapolation method using equation (3) has only four (two slopes for evaporation rates and two offsets). In addition, any inadequacy of the response model and empirical delay can lead to a "pull" on the underlying evaporation curve (figure 11). This additional freedom may be the reason why the resulting uncertainties for the droplet mass are significantly larger for the response fits. It also should be noted that the response model is not specifically designed to cover the filtering incurred with the Sensor mode

\footnotetext{
${ }^{1}$ In quoting minimum weights, manufacturers use a typical value rather than the specification value for the repeatability. In addition, the USP rule was changed in 2013 by reducing the coverage factor from 3 to 2 and the range available to rounding by changing the criterion from $0.1 \%$ to $0.10 \%$.
}
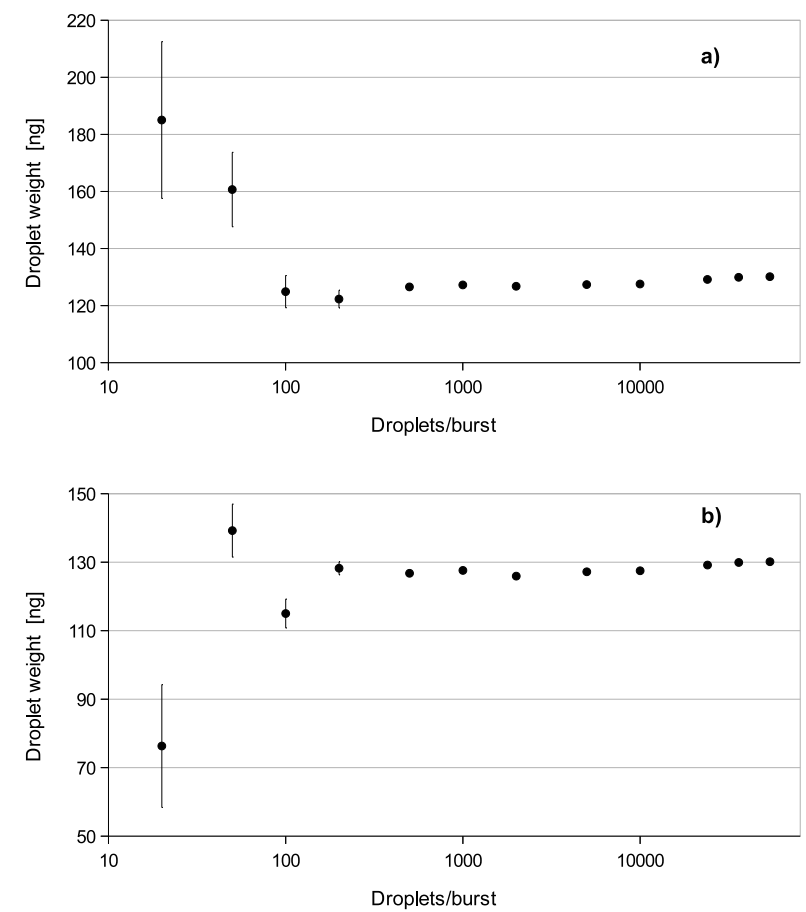

Figure 8: Averaged droplet masses from two-sided extrapolation versus aliquot size - a) Universal/Standard settings; b) Sensor/Unstable settings. The three data points at the right end are from the continuous mode measurements for 5000 droplets each bracketing the burst mode measurements.

of operating the XP2U microbalance. A modification to the exponential piece in equation (10) describing an underdamped oscillatory response [30] has been tried but did not give any consistent improvement.

A benefit of the response model is that it gives a fair estimate of the overall response time of the microbalance. Taking, somewhat arbitrarily, a saturation of $99 \%$ in equation (10) as the criterion, the fitted response constants (figure 12) of $\lambda=1.5 s^{-1}$ and $\lambda=0.3 s^{-1}$ yield response times of $3 s$ and $15 s$ for the two operating modes being considered. To this, the empirical delay (figure 13) needs to be added, which leaves the factory default mode essentially unchanged and lengthens the response of the filtered mode to $20 \mathrm{~s}$. For comparison, the extrapolation analysis was performed with a $15 s$ margin between the end of the dispensing and the beginning of the evaporation measurement after dispensing. Making some allowance for the slower response start than suggested by equation (10), the stick-andrelease behavior of the balance, and for the fluctuations in the fit results suggests margins of near $10 s$ for the factory default of the XP2U microbalance and $25 s$ for the Sensor/Unstable combination of settings. These results coincide quite well with the estimates gained from the static mass measurements.

Furthermore, the above analysis of the response behavior, in particular equation (12), shows that, for a weight changing linearly with time, the balance readings will follow that rate with a constant lag after the response time has expired. This ensures that the linear model applies to the evaporation rates without 

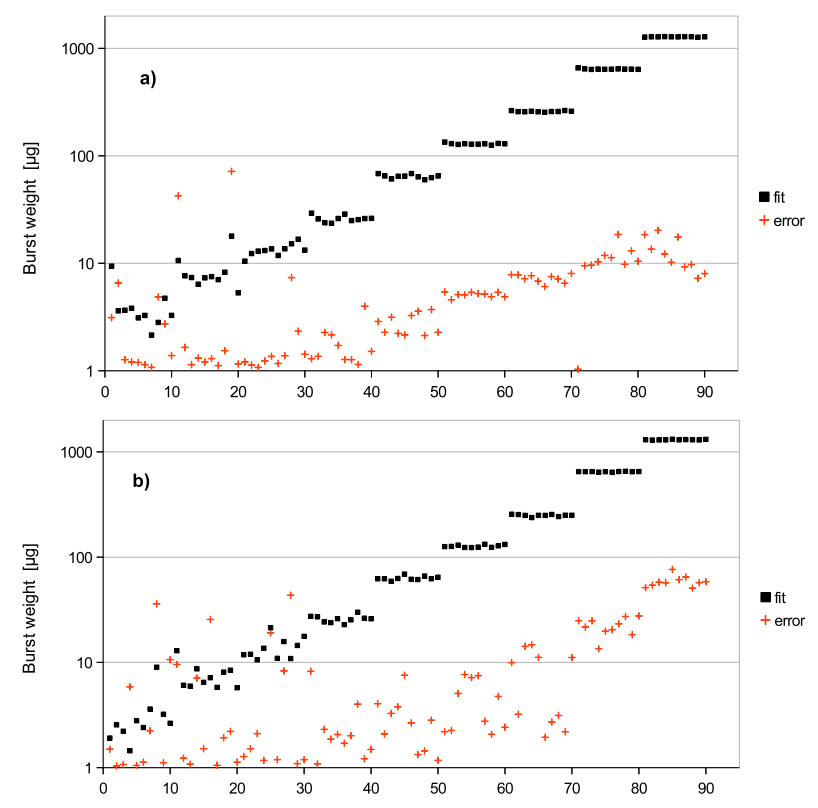

Figure 9: Raw burst weight data from response curve fitting sorted by aliquot size - a) Universal/Standard settings; b) Sensor/Unstable settings.

further response correction as well as to the continuous mode method of droplet mass measurement.

An additional item that the present data provide information on is the issue of refresh rate, or whether two consecutive weight values are independent measurements or the same single measurement picked up twice. Verkouteren and Verkouteren $[1,2]$ use $10-11 s$ intervals between data points to avoid any possible refresh delay, while in the present work a maximum update rate of near $0.2 s$ per data point is found and used. The manufacturer lists a refresh rate of $23 s^{-1}$, which indicates that the rate limit in the present work is specific to its data acquisition system. It should be noted that between these studies, different models of microbalances are used, and different and independent software programs to perform the data acquisition. The maximum-rate data available in the present case are those analyzed in real time around the bursts used for the cyclic measurements of various aliquot sizes (figures 7, 9). The data acquisition allows the tail evaporation data of one measurement to overlap the lead evaporation data for the next measurement. The available data (figure 14) thus contain four long blocks corresponding to the four sets of cycles through the 9 aliquots, the first two sets using the Sensor/Unstable settings and the other two the factory defaults. Figure 14 also shows read-out periods of constant consecutive values, with the longest such period being just under $5 s$ (close-up view in figure 15). A coarse distribution of the period lengths is given in table 8 .

The heavy filtering in the Sensor/Unstable configuration can be expected to lead to shorter and fewer periods of constant readout. This is indeed apparent in figure 14, with $9.3 \%$ of all periods having two or more consecutive constant values, while that fraction is $24.1 \%$ for factory default conditions. With 188 individual burst mode measurements contained in the data, each
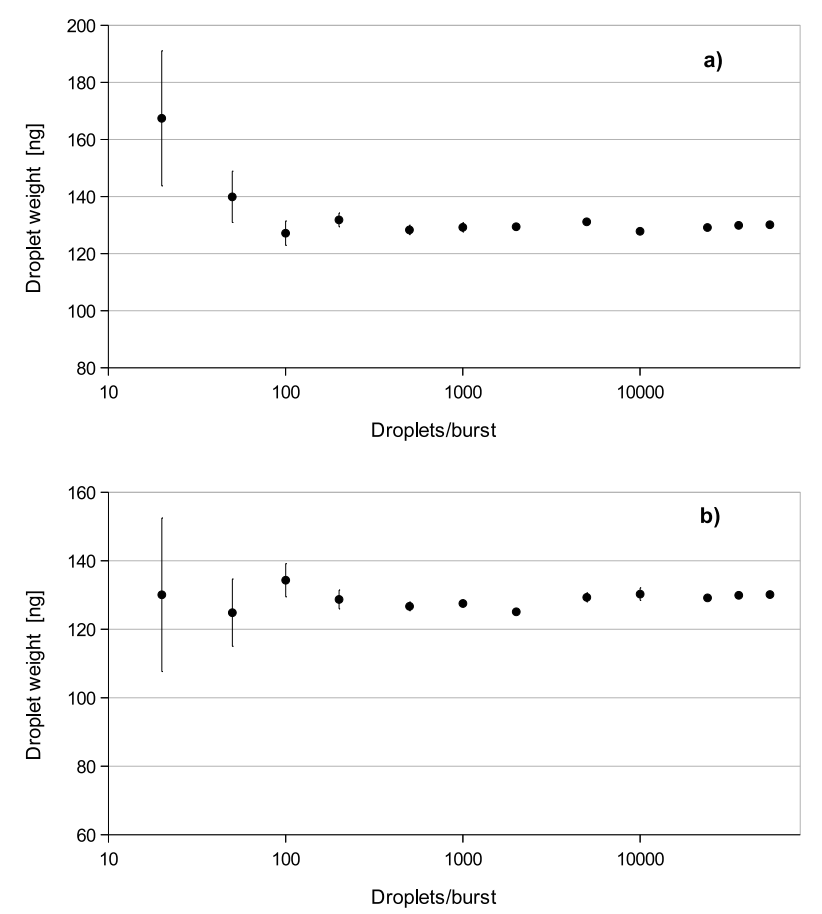

Figure 10: Averaged droplet masses from response curve fitting versus aliquot size - a) Universal/Standard settings; b) Sensor/Unstable settings. The three data points at the right end are from the continuous mode measurements for 5000 droplets each bracketing the burst mode measurements.

Table 8: Distribution of periods of constant weight - all data.

\begin{tabular}{lr}
\hline Length in $s$ & Occurrences \\
\hline$=0$ & 40476 \\
$0-1$ & 7503 \\
$1-2$ & 162 \\
$2-3$ & 22 \\
$3-4$ & 4 \\
$4-5$ & 1 \\
\hline
\end{tabular}

one will create a short period of constant readout values at the turn-over from pure evaporation to droplet dispensing and again at the end of the dispensing. These periods are typically well shorter than $1 s$. Longer periods are thus a feature of the response behavior of the balance. Detailed distributions on the basis of period lengths (figure 16) and of the counts of consecutive constant values (not shown, end point at 26 consecutive values), covering only the two long blocks of factory-default mode data, show a rapidly decaying shape. In the opinion of the author, they do not suggest that issues with updating the weight readings are prominent. Using some $20 s$ worth (or more) of consecutive data for each evaporation segment will be similarly little affected by the update behavior as the undersampling approach of using long intervals between data points.

An aspect not covered by the present measurements is the evaporation in flight. The issue is that the balance measures the droplet weight after a certain flight distance, but the weight at the time of drop formation is really the one of interest. This 

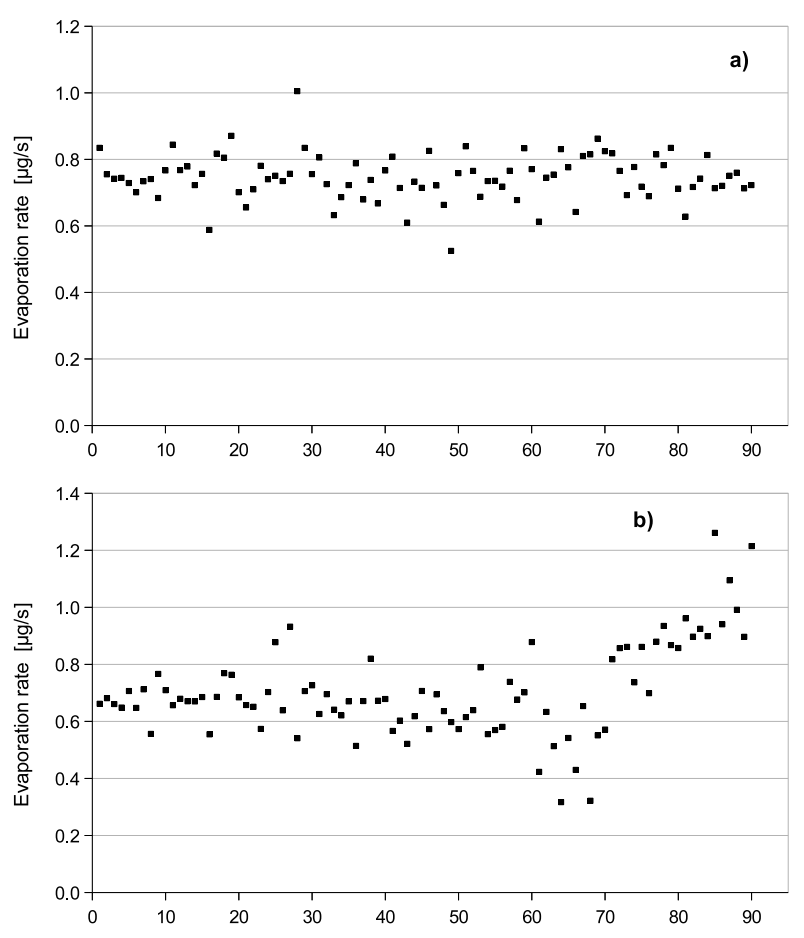

Figure 11: Evaporation rates from fits of equation (14) to all data - a) Universal/Standard settings; b) Sensor/Unstable settings. The data points correspond one-to-one to those in figure 9.

effect was noted by Verkouteren and Verkouteren [1] as having the potential to affect the droplet mass measurement on a scale comparable to or larger than the total measurement uncertainty. Their data (for isobutanol) show a clear effect when the droplets are allowed to travel through moistened but less than saturated air by keeping the dispensing orifice some distance above the nearly saturated open volume of their weighing boat, but are inconclusive for travel within the height of the boat. For the purpose of the present study, a rough estimate of the effect can be obtained numerically as follows. (Numerical values for the various parameters are collected in table S5 of the Supplementary Information.) The evaporation of small droplets can be described with a formula developed by Langmuir [31] with the mass change over time scaling with the radius. Rewriting this in terms of change of the surface area, the loss is a constant one over time (18),

$$
-\frac{d s}{d t}=\frac{8 \pi D m_{2} p_{0}}{\rho k_{B} T}
$$

with $s$ being the surface area of the droplet, $t$ the time, $D$ the diffusion coefficient for the vapor in air, $m_{2}$ the mass of one fluid molecule, $p_{0}$ the saturated vapor pressure for the fluid, $\rho$ the density of the fluid, $k_{B}$ the Boltzmann constant and $T$ the absolute temperature. A correction to equation (18) for the limit of very small radii proposed by Fuchs [32] turns out to be still negligible for the radii of interest here (order of $30 \mu \mathrm{m}$ ) [33]. Besides the explicit temperature term in the denominator, both $D$ and $p_{0}$ have significant temperature dependences, and $\rho$ a comparatively weaker one. The net effect is that with falling
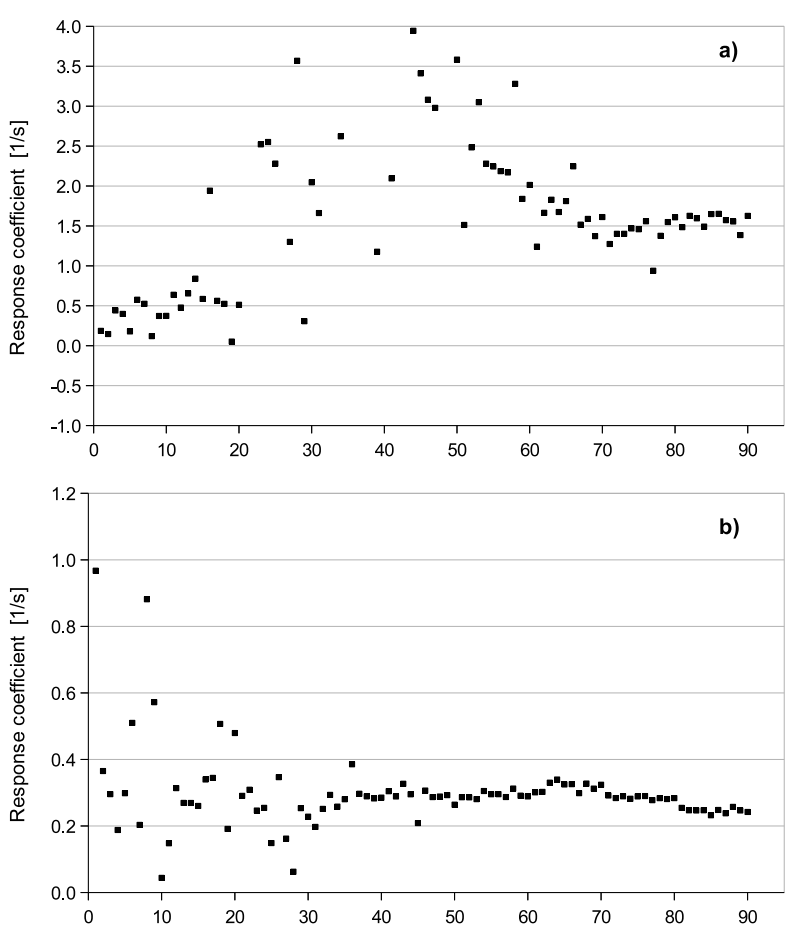

Figure 12: Response coefficients $\lambda$ from fits of equation (14) to all data - a) Universal/Standard settings; b) Sensor/Unstable settings. The data points correspond one-to-one to those in figure 9.

temperature the evaporation slows down. As evaporation entails cooling of the remaining fluid, the apparently constant rate of surface change gains a time dependence through the temperature drop. A detailed analysis is beyond the scope of the present work, but two simplified estimates give bounds for the actual effect. In integrating equation (18), the first estimate assumes for each time step an instant adjustment of the temperature of the whole droplet volume to make up for the energy lost to evaporation at the surface. This underestimates the temperature drop at the surface and thus overestimates the evaporation rate over time. The second estimate uses the diffusivity $\alpha$ to define a penetration depth (19) [34] as the thickness $d$ of a shell of the droplet providing all energy needed to sustain evaporation.

$$
d=\sqrt{\alpha t}
$$

The core of the droplet is left untouched, leading to an overestimate of the temperature drop near the surface and thus an underestimate of the evaporation rate. These two estimates are used to set the conservative bounds shown in table 9 for droplets of $30 \mu \mathrm{m}$ radius falling for up to $30 \mathrm{~ms}$. Due to air drag acting on the falling droplets, this is not too long a fall time to be considered in the standard setup on a jetlab 4 XLB $(28 \mathrm{~mm}$ height, $4 \mathrm{~m} / \mathrm{s}$ initial drop velocity, see figure 17). The drag effect calculation follows the work of Brown and Lawler [35], with their equation (19) used for the drag coefficient. (The importance of the drag effect in this context was pointed out previously by Verkouteren and Verkouteren [1].) Drop velocities in inkjet applications around $1.5-3 \mathrm{~m} / \mathrm{s}$ are not uncommon 

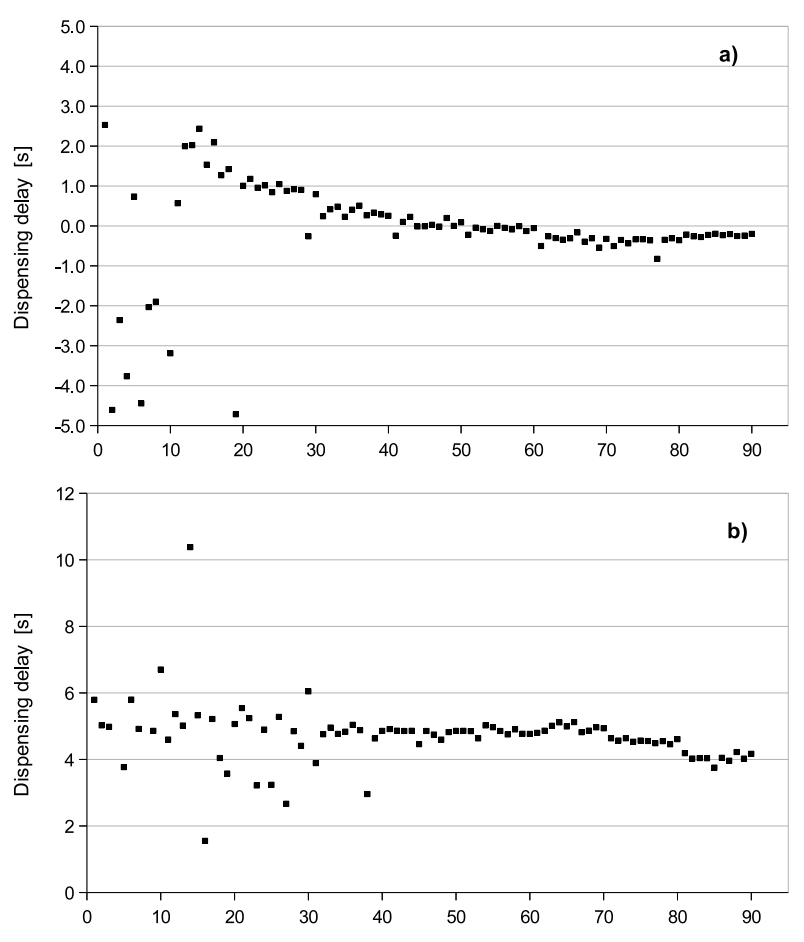

Figure 13: Delay values from fits of equation (14) to all data - a) Universal/Standard settings; b) Sensor/Unstable settings. The data points correspond one-to-one to those in figure 9.

$[1,2,12,27,36,37,38,39,40,41,42]$. The estimates in table 9 apply directly to droplets in dry air (nominally zero partial vapor pressure). The data of Verkouteren and Verkouteren (figure 6 in [1]) indicate an evaporation loss of order $0.5-1 \%$ for isobutanol droplets of $23 \mu \mathrm{m}$ radius at $>2 \mathrm{~ms}$ flight time in unsaturated air, in line with the present estimates. Their statistics and resolution appear insufficient to quantify any effect at higher saturation levels. The evaporation rates reported in table 6 indicate that the high level of saturation within the draft shield will reduce the evaporation rates by a factor 3-4. At that reduced level evaporation in flight should still be expected to be noticeable for volatile fluids and may need attention even

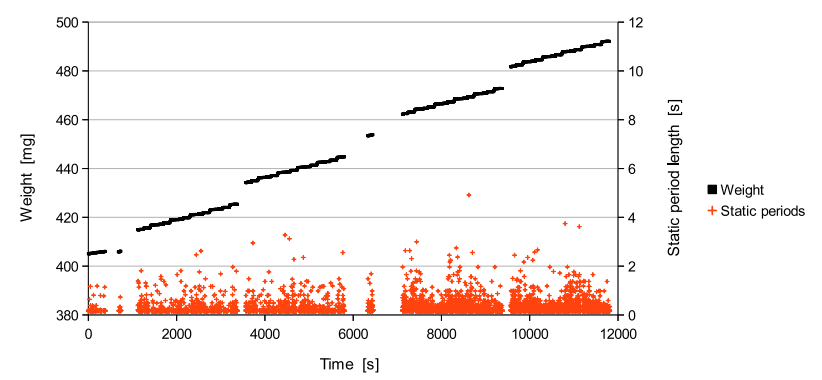

Figure 14: Weight data recorded over two sets of 10 cycles through 9 sample sizes. The lower, red data points represent the lengths of periods of constant weight, with single-value periods not included. The two sets are broken into two blocks each; the additional data served for operational verification.

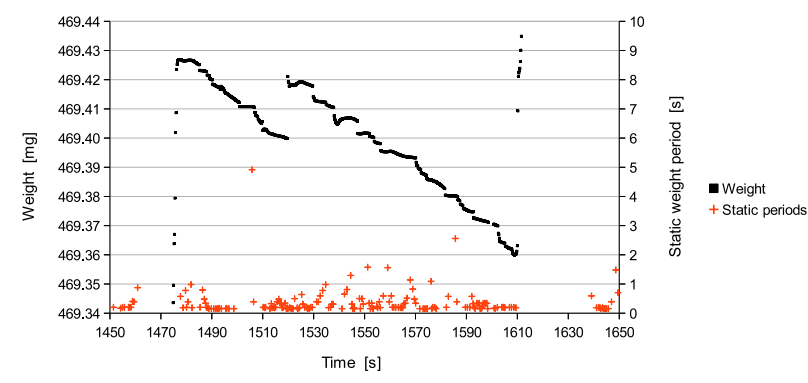

Figure 15: Narrow section of the factory default data clearly showing two static periods of $4.9 s$ at $\approx 1500 s$ and $2.5 s$ at $\approx 1580 s$. The time scale is reset from figure 14 and starts here at the beginning of the third long block of figure 14 . There are four dispense periods within the time window shown: 5000 droplets at $1475 s, 200$ droplets at $1520 s, 20$ droplets at $1565 s$, and 10000 droplets at $1620 \mathrm{~s}$.

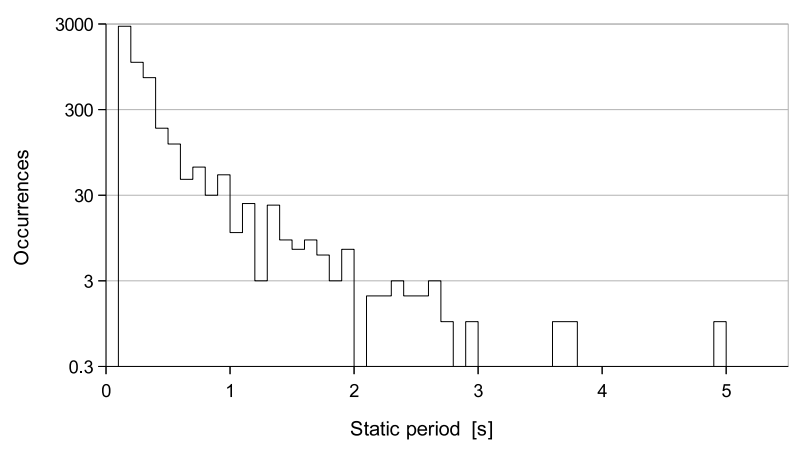

Figure 16: Distributions of weight repetitions under factory default conditions by period length. Data cover the two long blocks after $t=6000 \mathrm{~s}$ in figure 14 .

for fluids behaving similarly to water if high accuracy needs to be achieved and maintained. The droplet evaporation issue increases in importance with decreasing droplet radius. This issue needs further experimental work.

\section{Conclusions}

Using a microbalance to record a constantly changing weight as is done in inkjet gravimetry exposes some peculiar stickand-release behavior in the response of the balance, which must be accounted for in setting up the measurement and evaluation procedures. Tests with static calibration weights and the modeling of the response in inkjet gravimetry confirm that the balance response time for factory default settings remains within the factory specification, typically $10 s$ for the type of ultra microbalance considered here. Using the optional built-in filtering

Table 9: Estimated ranges of evaporation loss for droplets in flight (30 $\mu \mathrm{m}$ radius).

\begin{tabular}{lccc}
\hline Time of flight & Water & Ethanol & Isobutanol \\
\hline $10 \mathrm{~ms}$ & $0.81-1.48 \%$ & $1.38-2.02 \%$ & $0.78-1.39 \%$ \\
$20 \mathrm{~ms}$ & $1.08-2.39 \%$ & $1.64-3.62 \%$ & $1.06-2.40 \%$ \\
$30 \mathrm{~ms}$ & $1.24-3.06 \%$ & $1.79-4.94 \%$ & $1.23-3.20 \%$ \\
\hline
\end{tabular}




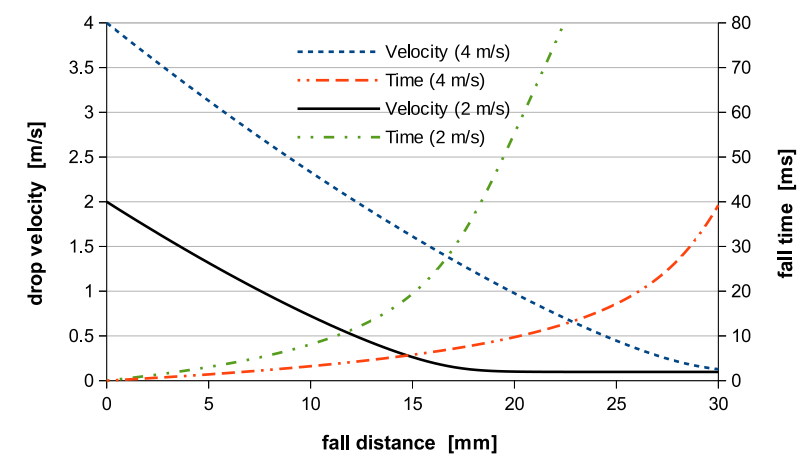

Figure 17: Velocity and fall time for a water droplet of $60 \mu \mathrm{m}$ diameter as a function of position along fall path for two initial drop velocities.

of the weight data to cope with the erratic behavior improves the measurements, at the expense of a longer effective response time. Still, the total time needed for a burst mode droplet mass measurement remains dominated by the time needed for acquiring clean evaporation data and dispensing the desired amount of droplets into the weighing boat. The evaporation periods should be a multiple of the longest refresh delays and the catchup times shown by the balance response. The evaporation out of a small weighing boat appears to behave quite similar to evaporation from a swimming pool. Air draft outside a draft shield does not cause any notable problems with the response of the balance and thus will not impair mass measurements as long as conditions remain stationary. For volatile fluids, evaporation in flight may be important and thus needs further experimental study.

\section{Acknowledgements}

The present work grew out of the check-out of jetlab 4 XLB print stations at MicroFab Technologies, Inc. Extensive feedback about the balance operation on those systems from Dr. Michael Verkouteren of NIST and discussions of various operational aspects with him are gratefully acknowledged.

[1] R.M. Verkouteren, J.R. Verkouteren, Inkjet metrology: High-accuracy mass measurements of microdroplets produced by a drop-on-demand dispenser, Anal. Chem. 81 (2009) 8577-8584.

[2] R.M. Verkouteren, J.R. Verkouteren. Inkjet metrology II: Resolved effects of ejection frequency, fluidic pressure, and droplet number on reproducible drop-on-demand dispensing, Langmuir 27 (2011) 9644-9653.

[3] K.-S. Kwon, D. Zhang, H.-S. Go, Jetting frequency and evaporation effects on the measurement accuracy of inkjet droplet amount, J. Imaging Sci. Technol. 59 (2015) 020401.

[4] T. Lindemann, Droplet generation from the nanoliter to the femtoliter range, Ph.D. Thesis, University of Freiburg, Freiburg, Germany, 2006, https://www.freidok.uni-freiburg.de/dnb/download/2798 (accessed 11 Feb. 2016).

[5] D. Liang, C. Steinert, S. Bammesberger, L. Tanguy, A. Ernst, R. Zengerle, P. Koltay, Novel gravimetric measurement technique for quantitative volume calibration in the sub-microliter range, Meas. Sci. Technol. 24 (2013) 025301.

[6] L. Tanguy, A. Ernst, S. Bammesberger, R. Zengerle, P. Koltay, Pressure driven and regulated dispenser for the microliter range. Proc. 1st Int. Conf. Microfluidic Handling Systems, 10-12 Oct 2012, Enschede, The
Netherlands, pp.50-53.

[7] S. Bammesberger, A. Ernst, L. Tanguy, R. Zengerle, P. Koltay, A calibration-free, disposable, non-contact reagent dosing cartridge for the sub- $\mu$ L range, Proc. 1st Int. Conf. Microfluidic Handling Systems, 10-12 Oct 2012, Enschede, The Netherlands, pp.54-57.

[8] S.B. Bammesberger, I. Malki, A. Ernst, R. Zengerle, P. Koltay, A calibration-free, noncontact, disposable liqud dispensing cartridge featuring an online process control, J. Lab. Autom. 19 (2014) 394-402.

[9] M. Verkouteren, G. Gillen, M. Staymates, J. Verkouteren, E. Windsor, M. Walker, C. Zeissler, M. Najarro, G. Klouda, T. Brewer, J. Staymates, R. Fletcher, J. Ott, T. Barr, S. Dickinson, J. Grander, M. Halter, H. Sievers, Ink jet metrology: New developments at NIST to produce test materials for security applications, Proc. NIP 27 and Digital Fabrication, 2-6 Oct 2011, Minneapolis, Minnesota, USA (Society for Imaging Science and Technology 2011) pp.705-708.

[10] D. Wallace, B. Antohe, Ink-jet technology for trace vapor detector development and calibration, PDHS S\&T/EXD Advanced Trace Detection Industry Day, 25-26 July 2013, Washington DC, http://www.microfab.com/images/papers/2013_07_26\%20 poster\%20for $\% 20$ dhs\%20industry\%20days.pdf (accessed 11 Feb 2016).

[11] J.R. Verkouteren, J. Lawrence, G.A. Klouda, M. Najarro, J. Grander, R.M. Verkouteren, S.J. York, Performance metrics based on signal intensity for ion mobility spectrometry-based explosive trace detectors using inkjet printed materials, Analyst 139 (2014) 5488-5498.

[12] G. Gillen, M. Najarro, S. Wight, M. Walker, J. Verkouteren, E. Windsor, T. Barr, M. Staymates, A. Urbas, Particle fabrication using inkjet printing onto hydrophobic surfaces for optimization and calibration of trace contraband detection sensors, Sensors 15 (2015) 29618-29634

[13] S. Muramoto, T.P. Forbes, A.C. van Asten, G. Gillen, Test sample for the spatially resolved quantification of illicit drugs on fingerprints using imaging mass spectrometry, Anal. Chem. 87 (2015) 5444-5450.

[14] C. Szakal, J.A. McCarthy, M.S. Ugelow, A.R. Konicek, K. Louis, B. Yezer, A.A. Herzing, R.J. Hamers, R.D. Holbrook, Preparation and measurement methods for studying nanoparticle aggregate surface chemistry, J. Environ. Monit. 14 (2012) 1914-1925.

[15] Y. Weng, H. Zeng, Y. Nakagawa, S. Ikeda, F. Chen, H. Nakajima, K. Uchiyama, Separation and determination of dopamine and epinephrine in serum by capillary electrophoresis with inkjet introduction system, Chromatography 34 (2013) 33-40.

[16] I.H. Xie, M.H. Wang, R. Carpenter, H.Y. Wu, Automated calibration of TECAN Genesis liquid handling workstation utilizing an online balance and density meter, Assay Drug Dev. Technol. 2 (2004) 71-80.

[17] D. Andersen, B. Rasmussen, K. Linnet, Validation of a fully automated robotic setup for preparation of whole blood samples for LC-MS toxicology analysis, J. Anal. Toxicology 36 (2012) 280-287.

[18] MicroFab Technologies, Inc., Jetlab 4xl-B product announcement, 2009, http://www.microfab.com/newssp-766878221/102-jetlab4xl-b-announcement http://www.microfab.com/index.php?option=com_content $\&$ view $=$ article $\&$ id=43:jetlab-xl-b\&catid=9: complete-systems \&Itemid=90 (accessed 10 Feb 2016).

[19] Joint Committee for Guides in Metrology, JCGM 100:2008: Evaluation of measurement data - Guide to the expression of uncertainty in measurement, 2008, http://www.bipm.org/ utils/common/documents/jcgm/JCGM_100_2008_E.pdf (accessed 12 Feb 2016).

[20] W. Funk, V. Dammann, G. Donnevert, Quality Assurance in Analytical Chemistry: Applications in Environmental, Food, and Materials Analysis, Biotechnology, and Medical Engineering, second ed., Wiley-VCH Verlag GmbH \& Co KGaA, Weinheim, Germany, 2007.

[21] G.A.F. Seber, C.J. Wild, Nonlinear Regression, John Wiley \& Sons, Hoboken, New Jersey, USA, 2003.

[22] K.W. Brodlie, A new direction set method for unconstrained minimization without evaluating derivatives, J. Inst. Maths. Applics. 15 (1975) 385-396.

[23] F. James, MINUIT Function minimization and error analysis, CERN Program Library D506, CERN, Geneva, 1994, https://root.cern.ch/sites/d35c7d8c.web.cern.ch/files/minuit.pdf (accessed 22 Nov 2016).

[24] W.H. Carrier, The temperature of evaporation, ASHVE Transactions 24 (1918) 25-50. 
[25] C.C. Smith, G. Löf, R. Jones, Measurement and analysis of evaporation from an inactive outdoor swimming pool, Solar Energy 53 (1994) 3-7.

[26] D. Liang, L. Tanguy, A. Ernst, R. Zengerle, P. Koltay, Novel gravimetric calibration method for nano liter liquid handling devices, Proc. 1st Int. Conf. Microfluidic. Handling Systems, 10-12 Oct 2012, Enschede, The Netherlands, pp.144-147.

[27] E. Windsor, M. Najarro, R. Bloom, B. Benner Jr., R. Fletcher, R. Lareau, G. Gillen, Application of inkjet printing technology to produce test materials of 1,3,5-trinitro-1,3,5 triazcyclohexane for trace explosive analysis, Anal. Chem. 82 (2010) 8519-8524.

[28] D.B. Wallace, Performance characteristics of a drop-on-demand ink jet device, Ph.D. Thesis, University of Texas at Arlington, Arlington, Texas, USA, 1987; see also: MicroFab Technote 99-04, Orifice diameter effects, 1999, http://www.microfab.com/images/pdfs/technote99-04.pdf (accessed 19 Jul 2016)

[29] United States Pharmacopeial Convention, Section 41 Balances, 2014, http://www.usp.org/sites/default /files/usp_pdf/EN/41_balances.pdf (accessed 22 Aug 2016)

[30] M. Alonzo, E.J. Finn, Physics, 2nd printing, Addison-Wesley Publ. Co., Reading, Massachusetts, USA, 1972, p.167.

[31] I. Langmuir, The evaporation of small spheres, Phys. Rev. 12 (1918) 368370.

[32] N.A. Fuchs, Über die Verdampfungsgeschwindigkeit kleiner Tröpfchen in einer Gasatmosphäre, Phys. Z. Sowjet. 6 (1934) 225-243.

[33] R.S. Bradley, M.G. Evans, R.W. Whytlaw-Gray, The rate of evaporation of droplets. Evaporation and diffusion coefficients, vapour pressures of dibutyl phthalate and butyl stearate, Proc. Roy. Soc. A164 (1945) 368 390.

[34] A. Bejan, Heat Transfer, John Wiley \& Sons, Inc., New York, New York, USA, 1993, p. 145.

[35] P.P. Brown, D.F. Lawler, Sphere drag and settling velocity revisited, J. Environ. Eng. 129 (2003) 222-231.

[36] J.J.P. Valeton, K. Hermans, C.W.M. Bastiaansen, D.J. Broer, J. Perelaer, U.S. Schubert, G.P. Crawford, P.J. Smith, Room temperature preparation of conductive silver features using spin-coating and inkjet printing, J. Mater. Chem. 20 (2010) 543-546.

[37] H.Y. Gan, X. Shan, T. Eriksson, B.K. Lok, Y.C. Lam, Reduction of droplet volume by controlling actuating waveforms in inkjet printing for micro-pattern formation, J. Micromech. Microeng. 19 (2009) 055010.

[38] K. A-Alamry, K. Nixon, R. Hindley, J.A. Odel, S.G. Yeates, Flowinduced polymer degradation during ink-jet printing, Macromol. Rapid Commun. 32 (2011) 316-320.

[39] J.S.R. Wheeler, S.W. Reynolds, S. Lancaster, V. Sanchez Romanguera, S.G. Yeates, Polymer degradation during continuous ink-jet printing, Polymer Degradation and Stability 105 (2014) 116-121.

[40] P.C. Duineveld, The stability of ink-jet printed lines of liquid with zero receding contact angle on a homogeneous substrate, J. Fluid Mech. 477 (2003) 175200

[41] E.L. Holthoff, M.E. Farrell, P.M. Pellegrino, Standardized sample preparation using a drop-on-demand printing platform Sensors 13 (2013) 5814-5825.

[42] C. Xu, W. Chai, Y. Huang, R.R. Markwald, Scaffold-free inkjet printing of three-dimensional zigzag cellular tubes, Biotechnol. Bioeng. 109 (2012) 3152-3160. 

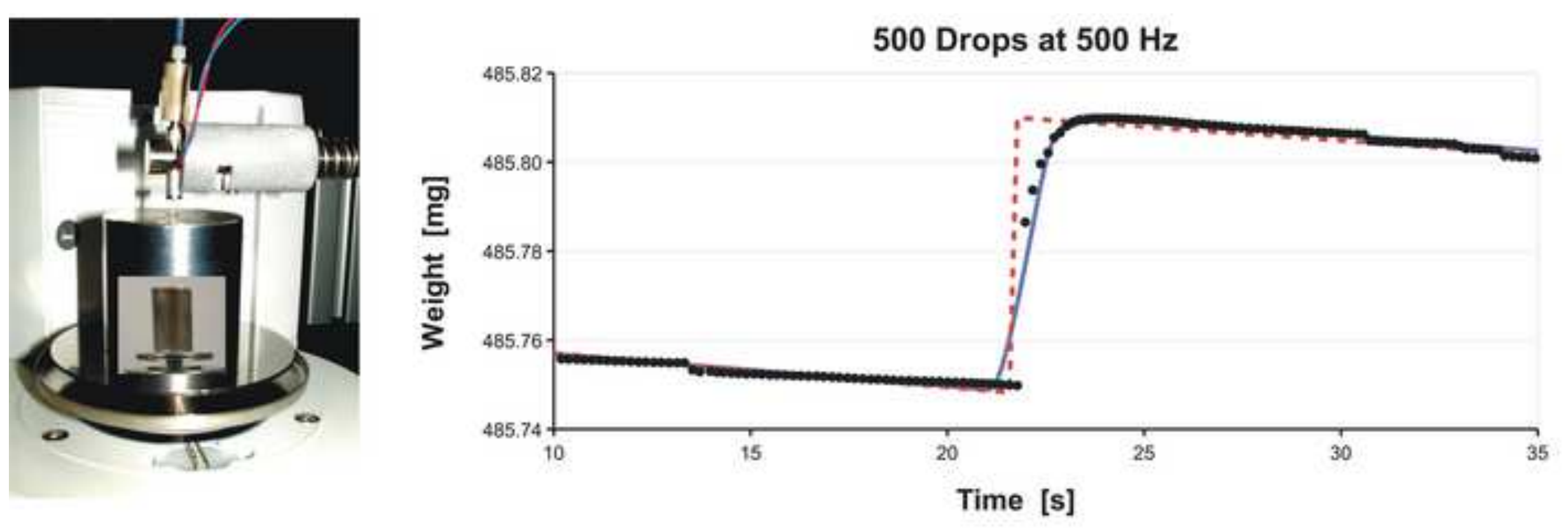

Look for: stick \& release, response time, evaporation.

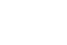

